\title{
Study on Handling Stability of AHVs Based on Closed-loop System
}

\author{
Lian $\mathrm{Xu}^{1, \mathrm{a}}$, Yang Sun ${ }^{1, \mathrm{~b}}$, Jie Tian ${ }^{1, \mathrm{c},{ }^{*}}$ and Ning Chen ${ }^{2, \mathrm{~d}}$ \\ ${ }^{1}$ College of Automobile and Traffic Engineering, Nanjing Forestry University, China \\ ${ }^{2}$ College of Mechanical and Electronic Engineering, Nanjing Forestry University, China \\ a287458924@qq.com, b809676898@qq.com, ǌtianjie@163.com, 'chennning@njfu.com.cn
}

\begin{abstract}
Keywords: AHV; handling stability; driver model; front-wheel steering; human-vehicle-road closed loop system

Abstract: When articulated heavy vehicles (AHV) are turning or under the action of lateral force, a serious swing and folding often occurs owing to the interaction between the tractor and the trailer. In order to solve this problem, the AHV is simplified into two parts, i.e., tractor and trailer, then the dynamics model is accordingly established, controller of the trailer active front-wheel steering (TAFS) is designed, single point preview driver model is combined, the closed-loop simulation model of driver-vehicle-road system is established, and the simulation is performed by the model based on MTLAB platform. The results show that the response of the AHV with the TAFS (AHV+TAFS) is superior to that of the ordinary AHV and the former's trailer has a good following performance.
\end{abstract}

\section{Introduction}

Articulated heavy vehicles (AHV) are popularly applied in the domestic and foreign highways, docks and port city roads because of its ability to load the overweight, super large or super long goods [1]. AHVs usually consist of a tractor or truck with at least a trailer. Different degrees of lateral swings often occur to the trailer while AHVs driving, which lead to the increase of the occupied lane width and affect the normal overtaking of the high-speed traffic flow, or even scratch the pedestrians or the vehicles on the inside of the corner and directly influence the traffic circulation [2]. Under the crosswind disturbance or obstacle avoidance under emergency, it is also prone to folding between the tractor and the trailer, leave the lane or rollover, which causes the very serious road safety problem [3]. Based on the single point preview driver model and the control strategy of the TAFS, human-vehicle-road closed-loop simulation system is established. And the simulation of the complex path is performed.

\section{AHV dynamics model}

In this paper, the AHV is composed of a tractor (with two axes) and a full trailer (also with two axes). And individual units are connected to each other by a hitch. Therefore the movement between the trailer and tractor is both independent and coupled to each other. And the vehicle dynamics model is shown in Fig.1.

Assuming $m_{1}$ and $m_{2}$ are the mass of the tractor and the trailer, $I_{\mathrm{Z} 1}$ and $I_{\mathrm{Z} 2}$ are the moment of inertia about $Z$ axis of the tractor and the trailer, $a_{1}$ and $b_{1}$ are the tractor's distance from the front and the rear axle to the center of gravity (CG), $a_{2}$ and $b_{2}$ are the trailer's distance from the front and rear axle to the $\mathrm{CG}, c_{1}$ and $c_{2}$ are the distance from the hitch point to the tractor's rear axle and the trailer's front axle, $\beta_{1}$ and $\beta_{2}$ are the sideslip angle of the tractor and the trailer, $\gamma_{1}$ and $\gamma_{2}$ are the yaw rate of the tractor and the trailer, $\delta_{1}$ and $\delta_{2}$ is the front wheel steering angle of the tractor and the trailer, $\Delta \varphi$ is the articulation angle, $k_{\mathrm{i}}$ is the tire cornering stiffness, $F_{Y i}$ is the lateral forces on the front and the rear tires of the tractor and the trailer $(i=1,2,3,4), F_{A X}$ and $F_{A Y}$ are the longitudinal and lateral force of the tractor at the hitch point, $F_{T X}$ and $F_{T Y}$ are the longitudinal and lateral force of the trailer at the hitch point. Based on the body fixed coordinate systems $x_{1}-y_{1}$ and $x_{2}-y_{2}$ for the tractor and the trailer, respectively, and assuming the front wheels of the trailer can also steer, the vehicle dynamic equations are as follows: 


$$
\left\{\begin{array}{l}
m_{1} u_{1}\left(r_{1}+\beta_{1}^{\mathcal{\alpha}}\right)=F_{Y 1} \cos \delta_{1}+F_{Y 2}+F_{A Y} \\
m_{2} u_{2}\left(r_{2}+\beta_{2}^{\alpha}\right)=F_{Y 3} \cos \delta_{2}+F_{Y 4}-F_{T y} \\
I_{Z 1} \hat{\alpha}=F_{Y 1} a_{1} \cos \delta_{1}-F_{Y 2} b_{1}-F_{A Y}\left(b_{1}+c_{1}\right) \\
I_{Z 2} \alpha=F_{Y 3} a_{2} \cos \delta_{2}-F_{Y 4} b_{2}-F_{T y} \cdot\left(a_{2}+c_{2}\right)
\end{array} .\right.
$$

Let $\mathbf{X}=\left[\begin{array}{llll}\beta_{1} & r_{1} & r_{2} & \Delta \varphi\end{array}\right]^{\mathrm{T}}$, the corresponding state equation is as follows:

$$
\begin{aligned}
& \mathbf{A} \mathbf{X}+\mathbf{B X}=\mathbf{C}_{1} \boldsymbol{\delta}_{1}+\mathbf{C}_{2} \boldsymbol{\delta}_{2} . \\
& \mathbf{A}=\left[\begin{array}{cccc}
a_{11} & a_{12} & 0 & 0 \\
a_{21} & a_{22} & a_{23} & a_{24} \\
a_{31} & 0 & a_{33} & 0 \\
0 & 0 & 0 & 1
\end{array}\right], \mathbf{B}=\left[\begin{array}{cccc}
b_{11} & b_{12} & 0 & 0 \\
b_{21} & b_{22} & b_{23} & b_{24} \\
b_{31} & b_{32} & b_{33} & b_{34} \\
0 & -1 & 1 & 0
\end{array}\right], \mathbf{C}_{1}=\left[\begin{array}{c}
c_{1} \\
c_{2} \\
c_{3} \\
0
\end{array}\right], \mathbf{C}_{2}=\left[\begin{array}{c}
0 \\
c_{4} \\
c_{5} \\
0
\end{array}\right] .
\end{aligned}
$$

where $a_{11}=m_{1} u\left(b_{1}+c\right), a_{12}=I_{z 1}, a_{21}=u\left(m_{1}+m_{2}\right) a_{21}=u\left(m_{1}+m_{2}\right), a_{22}=-m_{2}\left(b_{1}+c\right), a_{23}=-m_{2}\left(a_{2}+c_{2}\right)$, $a_{24}=m_{2} u, a_{31}=m_{1} u\left(a_{2}+c_{2}\right), a_{33}=I_{z 2}, b_{11}=k_{1}\left(a_{1}+b_{1}+c_{1}\right)+k_{2} c_{1}, b_{12}=\left[k_{1}\left(a_{1}+b_{1}+c_{1}\right) a_{1}-k_{2} b_{1} c\right] / u+m_{1} u\left(b_{1}+c_{1}\right)$ $b_{21}=k_{1}+k_{2}+k_{3}+k_{4} \quad, \quad b_{33}=\left[-k_{3} c_{2} a_{2}+k_{4}\left(a_{2}+b_{2}+c_{2}\right) b_{2}\right] / u \quad, \quad b_{22}=\left[k_{1} a_{1}-k_{2} b_{1}-\left(k_{3}+k_{4}\right)\left(b_{1}+c\right)\right] / u+m_{1} u ，$ $b_{23}=\left[-k_{3} c_{2}-k_{4}\left(a_{2}+b_{2}+c_{2}\right)\right] / u+m_{2} u \quad, \quad b_{24}=k_{3}+k_{4}, \quad b_{31}=\left(k_{1}+k_{2}\right)\left(a_{2}+c_{2}\right)+k_{3} a_{2}-k_{4} b_{2}, \quad b_{34}=k_{3} a_{2}-k_{4} b_{2}$, $b_{32}=\left[\left(a_{2}+c_{2}\right)\left(k_{1} a_{1}-k_{2} b_{1}\right)-\left(k_{3} a_{2}-k_{4} b_{2}\right)\left(b_{1}+c_{1}\right)\right] / u+m_{1} u\left(a_{2}+c_{2}\right), c_{1}=k_{1}\left(a_{1}+b_{1}+c_{1}\right), c_{2}=k_{1}, c_{3}=k_{1}\left(a_{2}+c_{2}\right)$, $c_{4}=k_{3} a_{2}, c_{5}=k_{3}$ 。

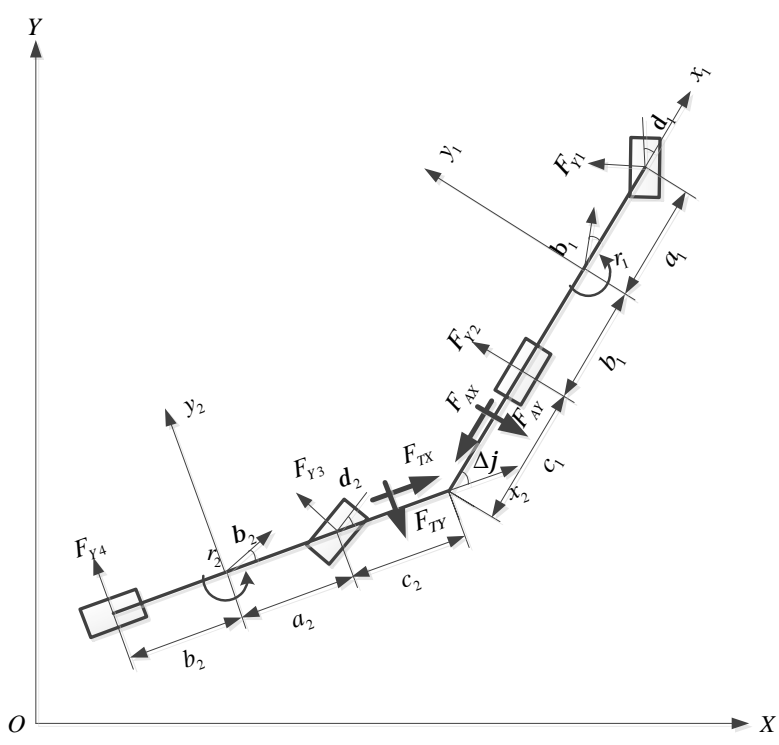

Fig.1 Vehicle dynamics model

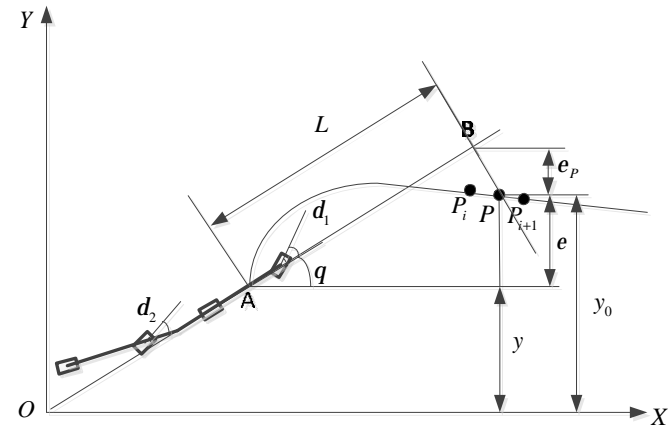

Fig.2 Single point preview model

\section{Establishment of driver model}

Since the middle of the last century, people have put forward preview models and compensation models based on all kinds of control theory [4-7]. We think that for the driver there is a relatively long reaction time at low speed so she/he can make a more ideal steering decision based on her/his experience. But at high speed, the driver's control behavior is more instinctive. At this time, the driver has to keep the vehicle travel by the preview track and also choose the larger turning radius to slow down the vehicle in turning. That is, the driver's driving experience is mainly reflected in the speed controlling and turning path choosing at high speed. The empirical control behavior, such as the speed controlling and the turning path selection, may not be taken into account because the main research object of the human-vehicle-road closed-loop simulation is the vehicle's kinematics and dynamics 
characteristics. So in this paper the single point preview driver model [5] is used, which is not only relatively simple, but also can reflect the handling stability of the vehicle.

\section{Single point preview driver model}

Visual information is the most important source of information for the driver, which is not the lateral deviation and azimuth deviation of the $\mathrm{CG}$, but the deviation value of the preview point $\mathrm{P}$ at the front of the vehicle. Fig. 2 shows the kinematic model of the single point preview and supposes the vehicle is moving along the flat curve (almost as a straight line), where y is the lateral displacement of the vehicle, $\theta$ is the vehicle yaw angle, $\mathrm{B}$ is the fixation point in front of the vehicle, $\mathrm{y}_{0}$ is the lateral displacement of the preview point $P$.

Assuming the driver's coordinate, $\mathrm{A}$, is $\left(x_{v}, y_{v}, \theta\right)$, the driver's eyes is focused on the point $\mathrm{B}\left(x_{f}, y_{f}\right)$, which is in the front of the vehicle. And the distance between $\mathrm{A}$ and $\mathrm{B}$ is $\mathrm{L}$, so $x_{f}=x_{v}+L \cos \theta$, $y_{f}=y_{v}+L \sin \theta$. Draw a straight line perpendicular to the line $\mathrm{AB}$ through point $\mathrm{B}$, which intersects the target path at preview point $\mathrm{P}$. In the Fig.2, $\mathrm{P}_{\mathrm{i}}\left(x_{i}, y_{i}\right)$ and $\mathrm{P}_{\mathrm{i}+1}\left(x_{i+1}, y_{i+1}\right)$ are two points before and after point $\mathrm{P}$ on the target path.

Deviation of point B to the target line is $\varepsilon_{p}=\varepsilon-L \theta$ because of $|\theta| \square$ 1.In addition $\Leftrightarrow u d y / d x=u \theta$, then $\varepsilon_{p}=\varepsilon-L d u$. The deviation of point $A$ to the target line is $\varepsilon=y_{0}-y$ and its change rate is $d \varepsilon / d t=-\delta$. According to the vehicle speed, the driver chooses the suitable preview aiming distance $\mathrm{L}$ in the reaction process, predicts the deviation of the vehicle to the ideal path and makes the necessary turning angle of the steering wheel to reduce the prediction deviation. The single point preview driver model is shown as Eq.3 [5-7], where $G$ is the closed loop gain, $G_{p}$ is the steering correction, $T_{s}$ is the combined delay time and $T_{p}$ is the driver's preview time.

$$
T_{s} \frac{d \delta_{f}}{d t}+\delta_{f}=G\left(y_{0}-y\right)+G_{p} \frac{d\left(y_{0}-y\right)}{d t} .
$$

Because $T_{p}=G_{p} / G=L / u, d \varepsilon / d t=-\oint$, Eq. 3 can be expressed as:

$$
\left\{\begin{array}{l}
\delta_{f}^{\mathcal{\alpha}}(t)=-\frac{1}{T_{s}} \delta_{f}(t)+\frac{1}{T_{s}} G \varepsilon_{p}(t) \\
\varepsilon_{p}=\varepsilon-L d u
\end{array} .\right.
$$

\section{Driver preview point}

Assuming the line equation perpendicular to the line $\mathrm{AB}$ through point $\mathrm{B}$ is as Eq.5, the line equation which through $\mathrm{P}_{\mathrm{i}}\left(x_{i}, y_{i}\right)$ and $\mathrm{P}_{\mathrm{i}+1}\left(x_{i+1}, y_{i+1}\right)$ is as Eq.6,

$$
\begin{aligned}
& \left(y_{f}-y_{v}\right)\left(y-y_{f}\right)+\left(x_{f}-x_{v}\right)\left(x-x_{f}\right)=0 . \\
& \left(x_{i+1}-x_{i}\right)\left(y-y_{i}\right)-\left(y_{i+1}-y_{i}\right)\left(x-x_{i}\right)=0 .
\end{aligned}
$$

Substituting $\mathrm{x}_{\mathrm{f}}$ and $\mathrm{y}_{\mathrm{f}}$ into Eq.5, then Eq.7 can be established by Eq.5 and 6:

$$
\left[\begin{array}{cc}
\cos \theta & \sin \theta \\
y_{i+1}-y_{i} & -x_{i+1}+x_{i}
\end{array}\right]\left(\begin{array}{l}
x \\
y
\end{array}\right)=\left(\begin{array}{c}
\left(x_{v}+L \cos \theta\right) \cos \theta+\left(y_{v}+L \sin \theta\right) \sin \theta \\
\left(y_{i+1}-y_{i}\right) x_{i}-\left(x_{i+1}-x_{i}\right) y_{i}
\end{array}\right) \text {. }
$$


It can be seen that it is not difficult to find the point $\left(x_{p}, y_{p}\right)$ from equation (7). If the point $\left(x_{p}, y_{p}\right)$ can meet the condition, $x_{i} \leq x_{p} \leq x_{i+1}$, then point $\left(x_{p}, y_{p}\right)$ is the preview point $\mathrm{P}$. If $x_{i}=x_{i+1}$, then the conditions will be $y_{i} \leq y_{p} \leq y_{i+1}$. If $\left(x_{p}, y_{p}\right)$ can not meet the conditions, then continue to find the preview point with $i$ increased by 1 until it satisfies the condition $\left(x_{i}, y_{i}\right) \leq\left(x_{p}, y_{p}\right) \leq\left(x_{i+1}, y_{i+1}\right)$. When get the preview point $\mathrm{P}$, the distance $\varepsilon_{p}$ is the preview deviation.

\section{Steering wheel turning direction}

After the determination of the preview point $\mathrm{P}$, it is still necessary to judge the direction of the steering wheel. Assuming point $\left(x_{i}, y_{i}\right)$ and point $\left(x_{i+1}, y_{i+1}\right)$ contribute to vector $\boldsymbol{a}$, point $\left(x_{i}, y_{i}\right)$ and point $\mathrm{B}$ contribute to vector $\boldsymbol{b}$, then the direction of the steering wheel can be judged by the positive and negative sign of the vector product $\boldsymbol{a} \times \dot{b}$.

\section{Controller of TAFS}

The folding and the transverse swing usually occur to AHVs due to the coupling of the tractor and the trailer. When the AHV turns, it is expected that both of the centroid trajectories of the tractor and the trailer are the same. Thus the CG of the tractor can be regarded as the preview point of the trailer front wheel and the articulation angle $\Delta \varphi$, which directly affects the trailer following performance, can be regarded as the preview deviation. According to this preview deviation, the trailer front wheel can reduce the deviation by turning a certain angle. Then according to the theory of single point preview, the control strategy of the trailer front wheel can be written as Eq.8, where $K_{s m}$ and $T_{s m}$ are the control gain and the delay time of the trailer steering mechanism.

$$
\dot{\delta_{3}}=-\frac{1}{T_{s m}} \delta_{3}+\frac{1}{T_{s m}} K_{s m} \Delta \varphi
$$

\section{Human-vehicle-road closed loop simulation}

The path used in the human-vehicle-road closed-loop simulation, such as serpentine road and lane road, are too simple and can not fully reflect the path trajectory and the vehicle response. In this paper a complex closed-loop path as shown in Fig. 3 is used, which includs all kinds of corners in the reality and has a strong research significance. By writing the relative programs, different curves shown in Fig.4-7 can be got.

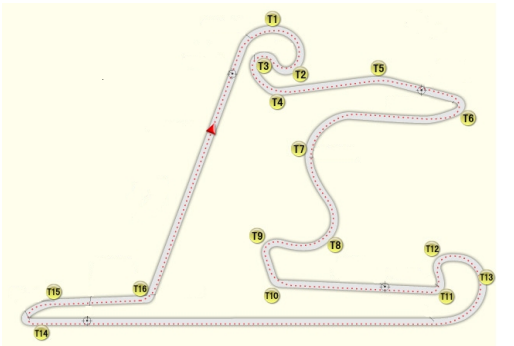

Fig.3 Complex closed-loop path

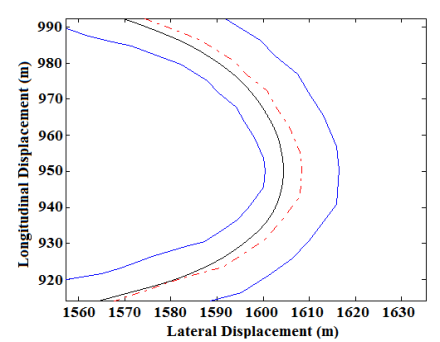

Fig.4 Trailer trajectory (AHV)

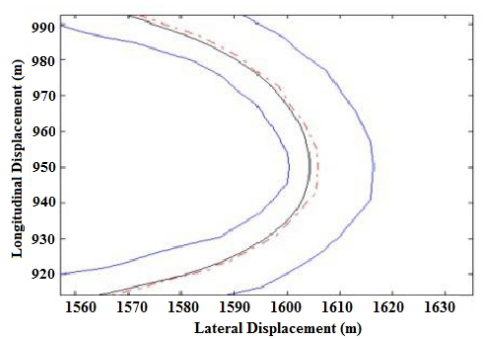

Fig.5 Trailer trajectory (AHV+TAFS)
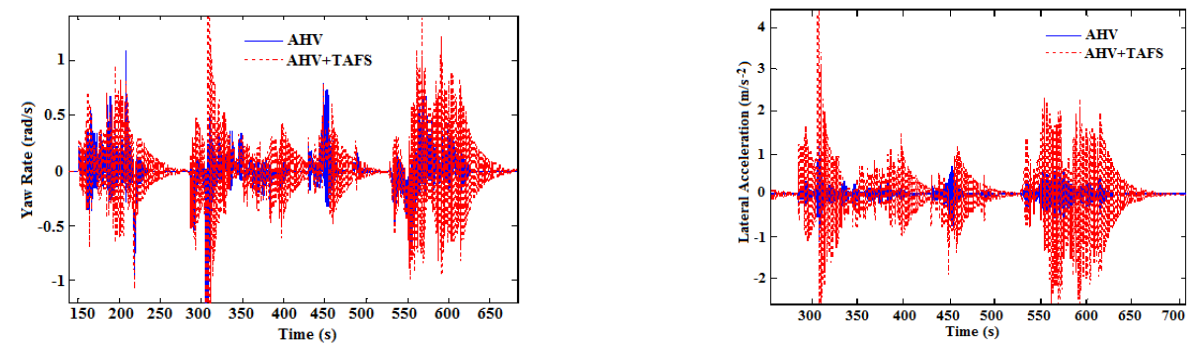

Fig.6 Yaw rates of AHV and AHV+TAFS Fig.7 Lateral accelerations of AHV and AHV+TAFS 
Fig. 4 and 5 are the partial enlarged details of the trailer trajectory of the AHV and AHV+TAFS. It can be drawn that the trailer trajectory of the AHV +TAFS is more close to the road center line and its path following is improved obviously.

Fig. 6 and 7 are the yaw rate and the lateral acceleration curve of the AHV and AHV+TAFS. It can be seen that the yaw rate and lateral acceleration changes with the bending degree of the road. The amplitudes of the AHV+TAFS are significantly smaller than those of the AHV. So the kinematic response and handling stability of the AHV+TAFS is better than the AHV.

\section{Conclusions}

1) The dynamic model of the AHV and the single point preview driver model are established, and the front-wheel-steering control strategy is designed based on the theory of preview theory.

2) The closed-loop simulation analysis of the AHV and AHV+TAFS are carried out. The results show that the dynamic performance and stability of the AHV+TAFS are better than the normal AHV.

\section{Acknowledgements}

This work was supported by Nanjing Forestry University Practice Innovation Training Program for Undergraduates(2015sjcx185), Jiangsu Government Scholarship for Overseas Studies (JS-2014-014), National Natural Science Foundation of China (11272159) and National Natural Science Foundation for youth of China (51305207).

\section{References}

[1] M. F.J. van de Molengraft-Luijten, I. J.M. Besselink , R. M.A.F. Verschuren and H. Nijmeijer: submitted to Vehicle System Dynamics (2012).

[2] Md. Manjurul Islam, X.J.Ding and Y.P.He: submitted to Vehicle System Dynamics, (2012).

[3] Md. Manjurul Islam, X.J.Ding and Y.P.He: submitted to International Journal of Heavy Vehicle Systems (2013).

[4] Y.Guan, Y.S.Chen, X.JIa, L.Z.Zhang, J.Zan: submitted to Automobile Engineering (2016) (In Chinese)

[5] A.J.Ma, J.G.Mao, H.Shen and H.Liu: submitted to Chinese Journal of Automobile Engineering (2013) (In Chinese)

[6] B.Yang, N.Chen, J.Tian and C.Q.Zhang: submitted to Mechanical Science and Technology for Aerospace Engineering (2014) (In Chinese).

[7] K.Jiang, M.Y.Zhang and Y.K.Chen: submitted to Journal of Traffic and Transportation Engineering (2015) (In Chinese). 